\title{
A branch of energetic-particle driven geodesic acoustic modes due to magnetic drift resonance
}

\author{
M. Sasaki ${ }^{1,2}$, N. Kasuya ${ }^{1,2}$, K. Itoh ${ }^{2,3}$, K. Hallatschek ${ }^{2,4}$, M. Lesur ${ }^{5}$, \\ Y. Kosuga ${ }^{2,6}$, and S.-I. Itoh ${ }^{1,2}$ \\ ${ }^{1}$ Research Institute for Applied Mechanics, Kyushu University, Kasuga 816-8580, Japan \\ ${ }^{2}$ Research Center for Plasma Turbulence, Kyushu University, Kasuga 816-8580, Japan \\ ${ }^{3}$ National Institute for Fusion Science, Toki 509-5292, Japan \\ ${ }^{4}$ Max-Planck-Institute for Plasma Physics, Boltzmannstr. 85748 Garching, Germany \\ ${ }^{5}$ Lorraine University, Institut Jean Lamour, Nancy 54-506, France \\ ${ }^{6}$ Institute for Advanced Study, Kyushu University, Fukuoka 812-8581, Japan
}

\begin{abstract}
Eigenmode analysis of geodesic acoustic modes (GAMs) driven by fast ions is performed, based on a set of gyrokinetic equations. Resonance to the magnetic drift of the fast ions can destabilize GAMs. A new branch is found in the family of GAMs, whose frequency is close to the magnetic drift frequency of the fast ions. The poloidal eigenfunction of this branch has bump structures in the poloidal direction where the resonance of the magnetic drift with the mode is strong. The ion heating rate by the GAMs is evaluated in the framework of quasi-linear theory. The heating is localized poloidally around the resonance locations. Owing to the bumps in the eigenfunction, the magnitude of the heating is much larger than that estimated without the magnetic drift resonance.
\end{abstract}

Keywords:Geodesic acoustic mode, GAM, EGAM, energetic particle, eigenfunction, eigenmode, energy channeling, ion heating

\section{Introduction}

The importance of geodesic acoustic modes (GAMs) on turbulent transport in magnetically confined plasmas has been recognized [1,2]. GAMs are driven by the coupling of micro-turbulence $[3,4,5]$, and/or by fast ions [6, 7, 8]. Experimental observations show that GAMs induced by fast ions (EGAMs) have much larger amplitude compared to those driven by turbulence $[9,10,11,12]$. Thus, impacts of EGAMs on bulk plasmas are expected to be significant. Numerical simulation reveals the nonlinear dynamics of 
EGAMs, such as coupling of EGAMs with turbulence [13], nonlinear saturation of EGAM [14], frequency chirping [15] and subcritical excitation of GAMs due to self-nonlinear coupling [16]. A process of energy channeling from energetic particles to bulk ions via GAMs has been proposed (GAM channeling) [17], and experimental validations are progressing $[18,19]$.

In the previous theory, resonance of GAMs with the transit motion of fast ions has been investigated. Two kinds of EGAMs have been reported; one whose frequency is the ordinary GAM frequency $\left(c_{s} / R, c_{s}\right.$ is the sound velocity, and $R$ is the major radius of plasma), and which is close to the transit frequency of fast ions $[6,20,21,22,23$, 24, 25, 26, 27]. Actually, several branches have been observed in experiments [11, 19]. The relation between the ordinary GAM branch and the EGAM has been studied, and a selection rule has been reported $[22,23,24]$. When the energy of fast ions becomes large, the magnetic drift motion of fast ions can exceed the effect of the transit motion. In such a situation, resonance due to the magnetic drift motion of fast ions should be considered.

In this study, we report an eigenmode analysis of a new (third) branch of GAMs driven by the resonance due to the magnetic drift motion of fast ions. The new branch has a frequency close to the magnetic drift frequency of fast ions. The eigenfrequency and the poloidal eigenfunction are obtained, based on gyrokinetic equation. The dispersion relation obtained in this paper includes the the contributions from modes with arbitrary poloidal mode numbers $m$, which is an extension of the previous studies where the included modes are truncated to keep only $m= \pm 1$ [22]. The poloidal eigenfunction of the new branch is shown to have bumps in the poloidal direction. The ion heating rate by the GAMs is evaluated in the framework of quasi-linear theory. The heating rate is shown to be localized poloidally around the resonance locations. The paper is organized as follows. A model for analyzing EGAMs is given in Sec. 2. In Sec. 3, the theoretical formulation of the dispersion relation is described, and the eigenfrequency and the eigenfunction are investigated. In Sec. 4, the ion heating rate by the GAMs is evaluated by using the obtained eigenfunctions within the framework of the quasi-linear theory. Summary is given in Sec. 5.

\section{Model}

We consider a simple tokamak equilibrium with circular magnetic surfaces. The equilibrium magnetic field is given by

$$
\boldsymbol{B}=\frac{B_{0}}{1+\epsilon \cos \theta}\left(\boldsymbol{e}_{\zeta}+\frac{\epsilon}{q} \boldsymbol{e}_{\theta}\right)
$$

where $\epsilon=r / R \ll 1$ is the inverse aspect ratio, $r$ and $R$ are the minor and major radii, respectively, and $q$ is the safety factor. The poloidal and toroidal angles are noted as $\theta$ and $\zeta$, and their unit vectors are $\boldsymbol{e}_{\theta}$ and $\boldsymbol{e}_{\zeta}$, respectively. We use the following normalization,

$$
t \rightarrow t \frac{v_{T}}{R}, v_{\perp} \rightarrow \frac{v_{\perp}}{v_{T}}, v_{\|} \rightarrow \frac{v_{\|}}{v_{T}}, k \rightarrow k \frac{v_{T}}{\Omega_{c i}}, \mathcal{E} \rightarrow \frac{\mathcal{E}}{T_{i}}, F_{j} \rightarrow \frac{F_{j}}{n_{0}}, \frac{e \phi}{T_{i}} \rightarrow \phi
$$


where $v_{T}=\sqrt{T_{i} / m_{i}}$ is the ion thermal velocity, $\Omega_{c i}$ is the ion cyclotron frequency, and $n_{0}$ is the background plasma density. The radial wavenumber of the GAM is expressed by $k, \mathcal{E}$ is the kinetic energy of particles, $F_{j}$ is the velocity distribution function of the mean component of the $j$-th particle speacies, and $\phi$ is the electrostatic potential perturbation of the GAM.

The perturbed velocity distribution function to the GAM fluctuations, $\delta f^{(j)}$, can be expressed as $\delta f^{(j)}=J_{0}\left(k v_{\perp}\right) \phi \frac{\partial F_{j}}{\partial \mathcal{E}}+g^{(j)}$, where $j$ represents the particle species, bulk ion or fast ions, and $J_{0}$ is the zeroth order Bessel function. Here the first term on the right hand side is the adiabatic response to potential fluctuations, and the second term is the non-adiabatic response. The non-adiabatic component $g^{(j)}$ is expressed as $g^{(j)}(\boldsymbol{v}, \boldsymbol{r})=$ $G_{\omega}^{(j)}(\boldsymbol{v}, \theta) e^{-i \omega t+i k r}+$ c.c., and $G_{\omega}^{(j)}$ satisfies a linear gyrokinetic equation [28],

$$
\left(\omega+i \omega_{t} \partial_{\theta}-\omega_{d} \sin \theta\right) G_{\omega}^{(j)}=-\omega J_{0}\left(k v_{\perp}\right) \phi_{\omega} \frac{\partial F_{j}}{\partial \mathcal{E}} .
$$

Here, the transit frequency is denoted by $\omega_{t}=v_{\|} / q$, and $\omega_{d}=k\left(v_{\|}^{2}+v_{\perp}{ }^{2} / 2\right)$ is the magnetic drift frequency associated with the geodesic curvature. Assuming adiabatic electrons, the quasi-neutrality condition is given as

$$
\sum_{j}\left\langle J_{0} G_{\omega}^{(j)}\right\rangle-\phi_{\omega}=\tau\left(\phi_{\omega}-\phi_{\omega, 0}\right)
$$

where the bracket $\langle\cdots\rangle=\int \cdots d^{3} v$ represents a velocity integral, $\tau=T_{i} / T_{e}$ is the normalized ion temperature, and $\phi_{\omega, 0}$ is the magnetic surface-averaged $\phi_{\omega}$. The detailed derivation of Eq. (3) is given in Appendix A. The properties of the new branch of EGAM is investigated from Eqs. (2) and (3) in the following sections.

The velocity distribution function of the bulk ions $F_{i}$ is assumed to be Maxwellian, $F_{i}=\pi^{-3 / 2} e^{-\mathcal{E}_{i}}$. For transparency of the analysis, the fast ions' distribution, $F_{h}$, is taken as

$$
F_{h}=\frac{n_{h}}{2 \pi u_{0}^{2}} \delta\left(u-u_{0}\right) \delta\left(\Lambda-\Lambda_{0}\right)
$$

where $n_{h}$ is the density of energetic particles normalized by $n_{0}, u_{0}$ is the speed of the fast ions normalized by the ion thermal velocity, and $\Lambda$ is the pitch angle defined as the parallel velocity normalized by the total velocity [27]. This distribution corresponds to the limit that the width of the pitch angle $\Delta \Lambda$ is much smaller than $\Lambda_{0}$. In reality, this condition corresponds to a case before the slowing down and the pitch angle scattering. We also assume that the fast ions are passing particles, holding the condition, $\Lambda_{0}>\sqrt{r / R}$.

\section{$3 \quad$ Eigenmode analysis}

In this section, a new branch of EGAMs is shown. Equation (2) has two kinds of resonances in accordance with the transit frequency and the drift frequency of the fast ions. Their frequencies are introduced as $\omega_{h} \equiv \omega_{t}\left(u_{0}, \Lambda_{0}\right), \omega_{D} \equiv \omega_{d}\left(u_{0}, \Lambda_{0}\right)$, which are functions of the velocity and the pitch angle of the fast ions. Thus, the dominant resonance depends 
on the velocity and the pitch angle of the fast ions. We can consider two limiting cases; (i): In the limit that the transit frequency of the fast ions is much larger than the drift frequency, $\omega_{h} \gg \omega_{D}$, the resonance due to the transit frequency becomes important, and the solution with $\omega \sim \omega_{h}$ appears $[6,7,22,24]$. (ii): In the limit that the resonance due to the drift frequency becomes dominant, $\omega_{h} \ll \omega_{D}$, a new solution with a frequency close to the drift frequency of the fast ions appears, $\omega \sim \omega_{D}$. We focus on the limiting case (ii) where the resonance due to the magnetic drift frequency becomes important. In the following, the eigenfrequency is assumed to satisfy the condition, $\omega \sim \omega_{G} \sim \omega_{D} \gg \omega_{h}$. The assumptions in this paper can be summarized by the conditions for the speed and pitch angle of the fast ions and for the radial wavenumber of the EGAM as

$$
\begin{aligned}
& u_{0} \gg\left|\frac{2 \Lambda_{0}}{k q\left(1+\Lambda_{0}^{2}\right)}\right|, \\
& \left|\Lambda_{0}\right|>\sqrt{r / R}, \\
& |k| \sim \frac{2 \omega_{G}}{u_{0}^{2}\left(1+\Lambda_{0}^{2}\right)} .
\end{aligned}
$$

We assume that the radial wavelength of the GAM is much larger than the ion gyroradius, and use an ordering in the small parameter $k \ll 1$. The resonance due to the magnetic drift frequency, $\omega_{D} \sin \theta$, is inhomogeneous in the poloidal direction, while the transit frequency is homogeneous. Thus, the poloidal inhomogeneity of the eigenfunction becomes prominent when the magnetic drift resonance is dominant, so that the mode truncation is not suitable for the new branch. The contributions from modes with arbitrary poloidal mode numbers are calculated without assuming the amplitude of the poloidal harmonics $\left|\phi_{m} / \phi_{0}\right| \sim O\left(k^{m}\right)$. This is an extension of previous studies where the eigenfunction is assumed to follow the above ordering, and is truncated to keep only $m= \pm 1[22,24]$.

\subsection{Derivation of the dispersion relation}

In this subsection, the dispersion relation of the EGAMs which includes the magnetic drift resonance is derived from Eq. (3). The velocity integrals of the responses of the bulk ions and of the fast ions to the GAM potential are calculated.

First, the response of the bulk ions to the GAM potential, $G_{\omega}^{(i)}$, is described. For the bulk ions, the transit frequency is assumed to be much larger than the magnetic drift frequency, $\omega_{t}\left(v_{T h}\right) \gg \omega_{d}\left(v_{T h}\right)$. Thus, only the resonance due to the transit frequency, which leads to the ion Landau damping, is considered for the bulk ions. The response $G_{\omega}^{(i)}$ is obtained from Eq. (2) as [28]

$$
G_{\omega}^{(i)}=J_{0} F_{i} \sum_{m=-\infty}^{\infty} \phi_{m} e^{i m \theta} \sum_{l=-\infty}^{\infty} C_{m, l} e^{i l \theta}
$$

where $C_{m, l}$ is expressed as

$$
C_{m, l}=\sum_{p=-\infty}^{\infty}(-1)^{p} i^{l} J_{p}(\delta) J_{l-p}(\delta) \frac{\omega}{\omega-(m+p) \omega_{t}} .
$$


Here, $\delta=\omega_{d} / \omega_{t}$ is an effect of the finite orbit width, which is treated as a smallness parameter. The coefficient $C_{m, l}$ has resonances with respect to the harmonics of the transit frequency, which corresponds to the Landau damping. It is noted that the order of $C_{m, l}$ follows $C_{m, l} \sim O\left(\delta^{l}\right)$. Here, we keep the terms up to $\delta^{2}$, and consider the contributions of modes with arbitrary poloidal mode numbers without assuming the ordering for $\phi_{m}$. In this approximation, the lowest order terms of the finite orbit width effect are included, which corresponds to the process of $\phi_{m}$ coupling to its side-band modes, $\phi_{m \pm 1}$. If one truncates the poloidal mode number at $m= \pm 1$, the previous theory is reproduced [22, 24]. The coefficient $C_{m, l}$ can be approximated as

$$
\begin{aligned}
& C_{m, l=0} \approx\left(1-\frac{\delta^{2}}{2}\right) \frac{\omega}{\omega-m \omega_{t}}+\frac{\delta^{2}}{4}\left\{\frac{\omega}{\omega-(m-1) \omega_{t}}+\frac{\omega}{\omega-(m+1) \omega_{t}}\right\}, \\
& C_{m, l= \pm 1} \approx i \frac{\delta}{2}\left\{\frac{\omega}{\omega-m \omega_{t}}-\frac{\omega}{\omega-(m \pm 1) \omega_{t}}\right\} .
\end{aligned}
$$

The order of $C_{m,|l|>1}$ in $\delta$ is higher than $\delta^{2}$, which we neglect. The velocity integral of the response of the bulk ions, Eq. (8), is calculated as

$$
\left\langle J_{0} G_{\omega}^{(i)}\right\rangle=\sum_{m=-\infty}^{\infty} \phi_{m} e^{i m \theta} \sum_{l=-\infty}^{\infty} I_{m, l} e^{i l \theta},
$$

where $I_{m, l}$ is defined as

$$
\begin{aligned}
I_{m, l=0} & =\left\langle J_{0}^{2} F_{i} C_{m, l=0}\right\rangle \\
& =-\left(1-\frac{k^{2}}{2}\right) \frac{q \omega}{m} Z\left(\frac{q \omega}{m}\right)-\frac{k^{2} q^{2}}{4}\left(\alpha_{m-1}+\alpha_{m+1}-2 \alpha_{m}\right), \\
I_{m, l= \pm 1} & =\left\langle J_{0}^{2} F_{i} C_{m, l= \pm 1}\right\rangle \\
& =i \frac{k q}{2}\left(\beta_{m \pm 1}-\beta_{m}\right),
\end{aligned}
$$

and $I_{m,|l|>1}$ is defined as zero. Here, $Z(x)$ is the plasma dispersion function. The functions $\alpha_{m}$ and $\beta_{m}$ for $m \neq 0$ are introduced as

$$
\begin{aligned}
& \alpha_{m}=\left(\frac{q \omega}{m}\right)^{2}+\left\{\left(\frac{q \omega}{m}\right)^{3}+\frac{q \omega}{m}+\frac{m}{2 q \omega}\right\} Z\left(\frac{q \omega}{m}\right), \\
& \beta_{m}=\frac{q \omega}{m}+\left\{\left(\frac{q \omega}{m}\right)^{2}+\frac{1}{2}\right\} Z\left(\frac{q \omega}{m}\right) .
\end{aligned}
$$

These functions at $m=0$ are defined as $\alpha_{m=0}=-3 / 2$ and $\beta_{m=0}=0$.

Next, the response of the fast ions to the GAM potential, $G_{\omega}^{(h)}$, is described. For the fast ions, the magnetic drift frequency is assumed to be much larger than the transit frequency, $\omega_{D} \gg \omega_{h}$. For the transparency of the argument, the resonance of the transit 
frequency is neglected, and only the resonance due to the magnetic drift frequency is considered. In this limit, the response $G_{\omega}^{(h)}$ can be obtained from Eq. (2) as

$$
G_{\omega}^{(h)}=-\frac{\omega}{\omega-\omega_{d} \sin \theta} J_{0} \frac{\partial F_{h}}{\partial \mathcal{E}} \phi_{\omega}(\theta) .
$$

The response function of the fast ions features a resonance due to the magnetic drift. This resonance gives the new unstable branch. Keeping the resonance due to the magnetic drift, the velocity integral of Eq. (14) is obtained as

$$
\begin{aligned}
& \left\langle J_{0} G_{\omega}^{(h)}\right\rangle=\sum_{m=-\infty}^{\infty} \phi_{m} e^{i m \theta} \sum_{l=-\infty}^{\infty} H_{l} e^{i l \theta}, \\
& H_{l}=\oint 2 n_{h} J_{0}\left(k v_{\perp, h}\right)^{2} k \frac{\omega \sin \theta e^{-i l \theta}}{\left(\omega-\omega_{D} \sin \theta\right)^{2}} \frac{d \theta}{2 \pi} .
\end{aligned}
$$

The integral $H_{l}$ resonates at $\omega=\omega_{D} \sin \theta$, which gives an unstable branch of the EGAM.

The eigenequation is derived by substituting Eqs. (11) and (15a) into Eq. (3) as

$$
\sum_{m=-\infty}^{\infty}\left[\tau+1-\sum_{l=-\infty}^{\infty}\left(H_{l}+I_{m, l}\right) e^{i l \theta}\right] \phi_{m} e^{i m \theta}-\tau \phi_{\omega, 0}=0 .
$$

Equation (16) can be rewritten in a matrix form as

$$
\begin{aligned}
& \sum_{\nu=-\infty}^{\infty} D_{\mu, \nu} \phi_{\nu}=0, \\
& D_{\mu, \nu}=\tau \delta_{\mu, \nu}\left(1-\delta_{\mu, 0}\right)-\delta_{\mu, \nu}-\left(H_{\mu-\nu}+I_{\nu, \mu-\nu}\right),
\end{aligned}
$$

where $\mu$ is an integer $(\mu=-\infty, \cdots, \infty)$, and $\delta_{\mu, \nu}$ is the Kronecker delta. The first term in Eq. (17b) stems from the electron dynamics, the second term is the contribution of the adiabatic response of the bulk ions, and the third term with $I_{\nu, \mu-\nu}$ and $H_{\mu-\nu}$ come from the non-adiabatic responses of the bulk and the fast ions, respectively. The magnetic drift resonance and the Landau damping are included self-consistently, which are included in $H_{\mu-\nu}$ and $I_{\nu, \mu-\nu}$ respectively. The dispersion relation is obtained as

$$
\operatorname{det} D_{\mu, \nu}=0 \text {. }
$$

If one truncates the poloidal mode number at $|m|=1$, the dispersion relation without the contribution of the fast ions reproduces the results reported in [28, 29]; solutions with the ordinary GAM frequency and the ion sound wave branches are obtained. Here, the ordinary GAM frequency, $\omega_{G}$, is approximated as

$$
\omega_{G}=\sqrt{\left(\frac{7}{4}+\frac{1}{\tau}\right)\left[1+\frac{46 \tau^{2}+32 \tau+8}{(7 \tau+4)^{2} q^{2}}\right]},
$$

in the large safety factor limit [28]. 


\subsection{Eigenfrequency and eigenfunction}

The dispersion relation, Eq. (18), and the eigenequation, Eq. (17a) are solved numerically, and the eigenfrequency and eigenfunctions are described in this section.

Figure 1 illustrates the eigenfrequency as a function of the velocity of the fast ions. The real part of the eigenfrequency has solutions with Rew $\approx \omega_{G}, \omega_{D}$. The solution with the magnetic drift frequency appears owing to the magnetic drift resonance, which is the new solution in the family of the GAMs. The frequency of the unstable branch is close to $\omega_{D}$ when $\omega_{D}<\omega_{G}$, and it converges to $\omega_{G}$ when $\omega_{D}>\omega_{G}$. The frequency of the stable branch changes from $\omega_{G}$ to $\omega_{D}$, with the increase of the fast ions. The growth rate of the unstable branch has a maximum value at $\omega_{D}=\omega_{G}$. The GAMs are found to be destabilized by the magnetic drift resonance of the fast ions. It should be noted that, in the previous studies, the effect of the magnetic drift of the fast ions is included perturbatively to modify the resonance due to the transit motion of the fast ions, and the magnetic drift resonance is not considered. The parameter dependence of the eigenfrequency of the branch with $\omega \sim \omega_{G}$ is discussed in Appendix B.

The poloidal eigenfunction is calculated by $\phi_{\omega}=\sum_{m} \phi_{m} e^{i m \theta}$, where $\phi_{m}$ is obtained from the eigenequation Eq. (17a). The poloidal eigenfunction of the electrostatic potential is shown in Fig. 2(a). The real part of the eigenfunction has bumps where the magnetic drift resonance is satisfied, in addition to the $\sin \theta$ dependence as is predicted by the previous studies. The imaginary part of the eigenfunction appears near the resonance locations, which indicates a phase shift. The characteristic features such as the bump structures and the phase shift exist in the upper part of the poloidal cross section because positive $k_{r}$ is assumed. In the case of negative $k_{r}$, the characteristic features appears in the bottom part of the poloidal cross section. The poloidal mode spectrum of the eigenfunction is shown in Fig. 2(b). The eigenfunction has much larger poloidal harmonics than that assumed in the previous studies as $\left|\phi_{m}\right| / \phi_{0} \sim k^{m}$, which is owing to the bump structures of the eigenfunction. The distance between the resonant locations corresponds to $m \approx 3$ in the case of Fig. 2(a), so that there is a peak at $m=3$ in 2(b). The poloidal structure of the density fluctuations of the electrons, the bulk ions and the fast ions can be calculated as

$$
\begin{aligned}
& n_{\omega}^{(e)}=\tau\left(\phi_{\omega}-\phi_{\omega, 0}\right), \\
& n_{\omega}^{(i)}=-\phi_{\omega}+\left\langle J_{0} G_{\omega}^{(i)}\right\rangle, \\
& n_{\omega}^{(h)}=\left\langle J_{0} G_{\omega}^{(h)}\right\rangle .
\end{aligned}
$$

As is shown in Fig. 3 (a), the electron density fluctuation has bumps similar to those in the electrostatic potential, and bumps for the fast ions are localized near the resonance locations. The time evolutions of the density fluctuations are shown in Fig. 3 (b)-(d), where the time evolution is calculated by $\operatorname{Re}\left[n_{\omega}^{(j)} e^{-i \omega t}\right]$. The phase shift can be seen near the resonance locations. 

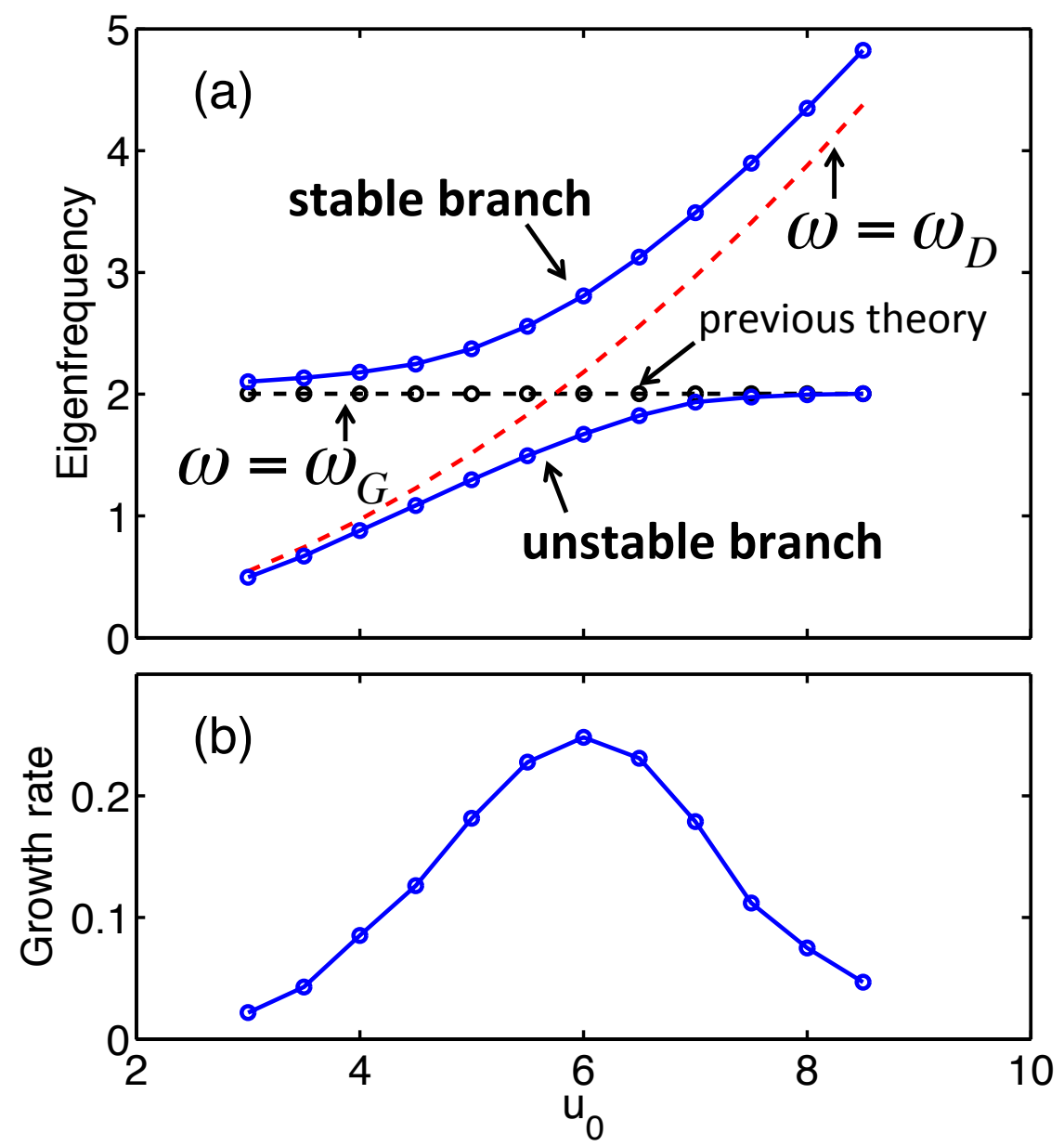

Fig. 1: Dependence of (a) eigenfrequency and (b) growth rate against the velocity of fast ions. The calculation is performed by using the following set of parameters; $q=3, \tau=$ $0.5, k_{r}=0.12, n_{h}=0.015, \Lambda_{0}=0.1$. 

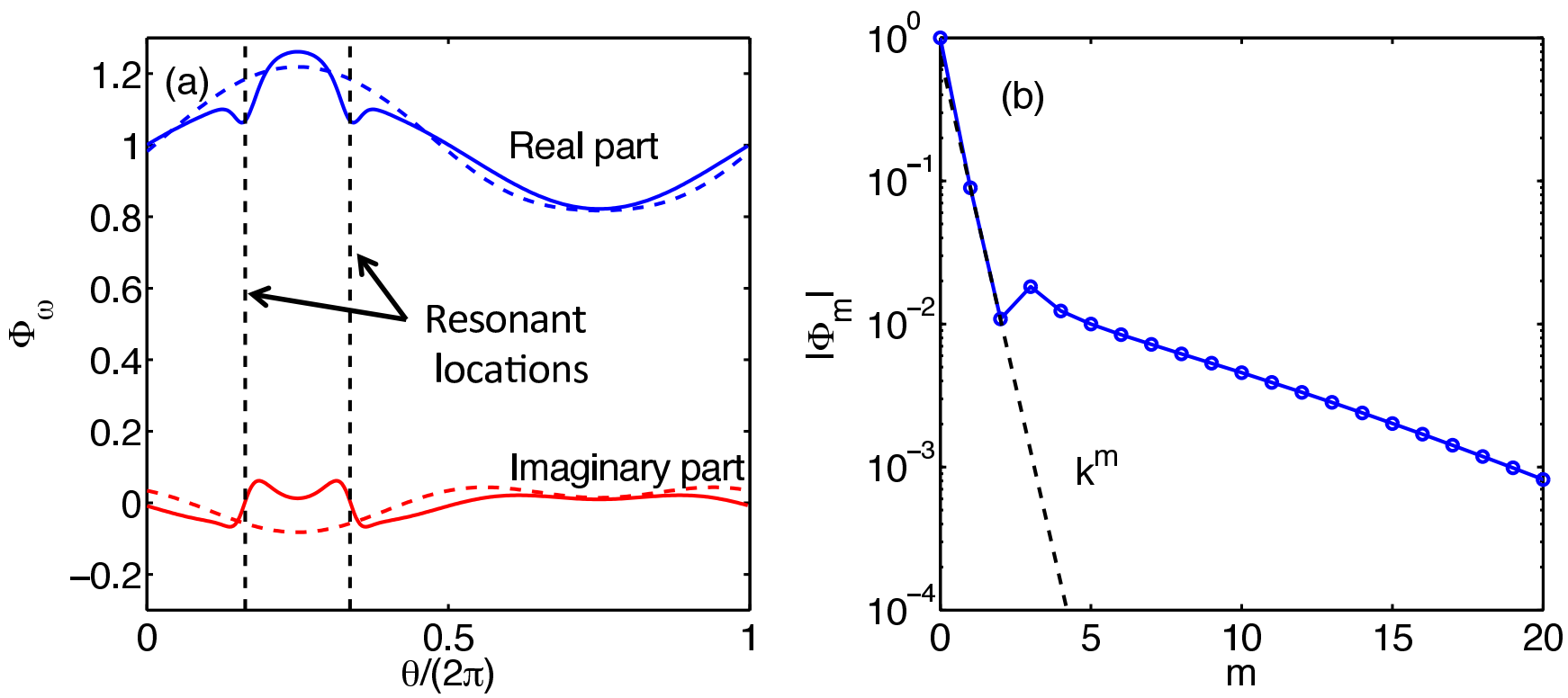

Fig. 2: (a) Poloidal eigenfunction in the case of $q=3, \tau=0.5, k_{r}=0.12, n_{h}=0.015, \Lambda_{0}=$ $0.1, u_{0}=5$. The eigenfunction without the energetic particle effect is also shown as a dashed curve. The eigenfunction has bumps and the imaginary part appears near the resonance locations. (b) Poloidal mode decomposition of eigenfunction. The dotted line shows $k^{m}$, which corresponds to amplitudes of the poloidal modes assumed in previous studies.

\section{Discussions on impacts on background plasmas}

In this section, effects of the $\omega_{D}$-branch on the background plasmas such as ion heating and turbulence are discussed.

The GAMs heat the bulk ions through the ion Landau damping. The ion heating rate, $P$, is evaluated within the framework of the quasi-linear theory. By using the eigenfunctions obtained above, $P$ is expressed by the product of the electric field, $\boldsymbol{E}_{\omega}$, with the current, $\boldsymbol{J}_{\omega}$, as

$$
\begin{aligned}
P & =\operatorname{Re}\left[\boldsymbol{E}_{\omega} \cdot \boldsymbol{J}_{\omega}^{*}+\boldsymbol{E}_{\omega}^{*} \cdot \boldsymbol{J}_{\omega}\right], \\
& =-2 \operatorname{Re}\left[\left\langle\omega_{t} \delta f_{\omega}^{(i)}\right\rangle \partial_{\theta} \phi_{\omega}^{*}\right]-2 \operatorname{Im}\left[\left\langle\omega_{d} \delta f_{\omega}^{(i)}\right\rangle \phi_{\omega}^{*} \sin \theta\right] .
\end{aligned}
$$

The poloidal structure of $P$ is illustrated in Fig. 4. The dotted line in Fig. 4 shows the heating rate evaluated without the deformation due to the magnetic drift resonance. The localized ion heating around the resonance locations is owing to the bump structures of the eigenfunction. The poloidal angle averaged heating rate which is normalized by the energy loss of the bulk plasma, $W_{p} \tau_{E}^{-1}$, can be estimated as $\oint P d \theta /(2 \pi)\left(W_{p} \tau_{E}^{-1}\right)^{-1}=0.37$ when $\tau_{E}=100$, where $W_{p}$ is the stored energy and $\tau_{E}$ is the energy confinement time normalized by $v_{T} / R$. In this manner, the ion heating effect of a large amplitude GAM can not be neglected with respect to the total energy balance of the bulk plasmas.

The $\omega_{D}$-branch accompanies a sheared poloidal flow with similar to the standard GAM, which has a suppression effect on turbulence. One of characteristics of the branch with 

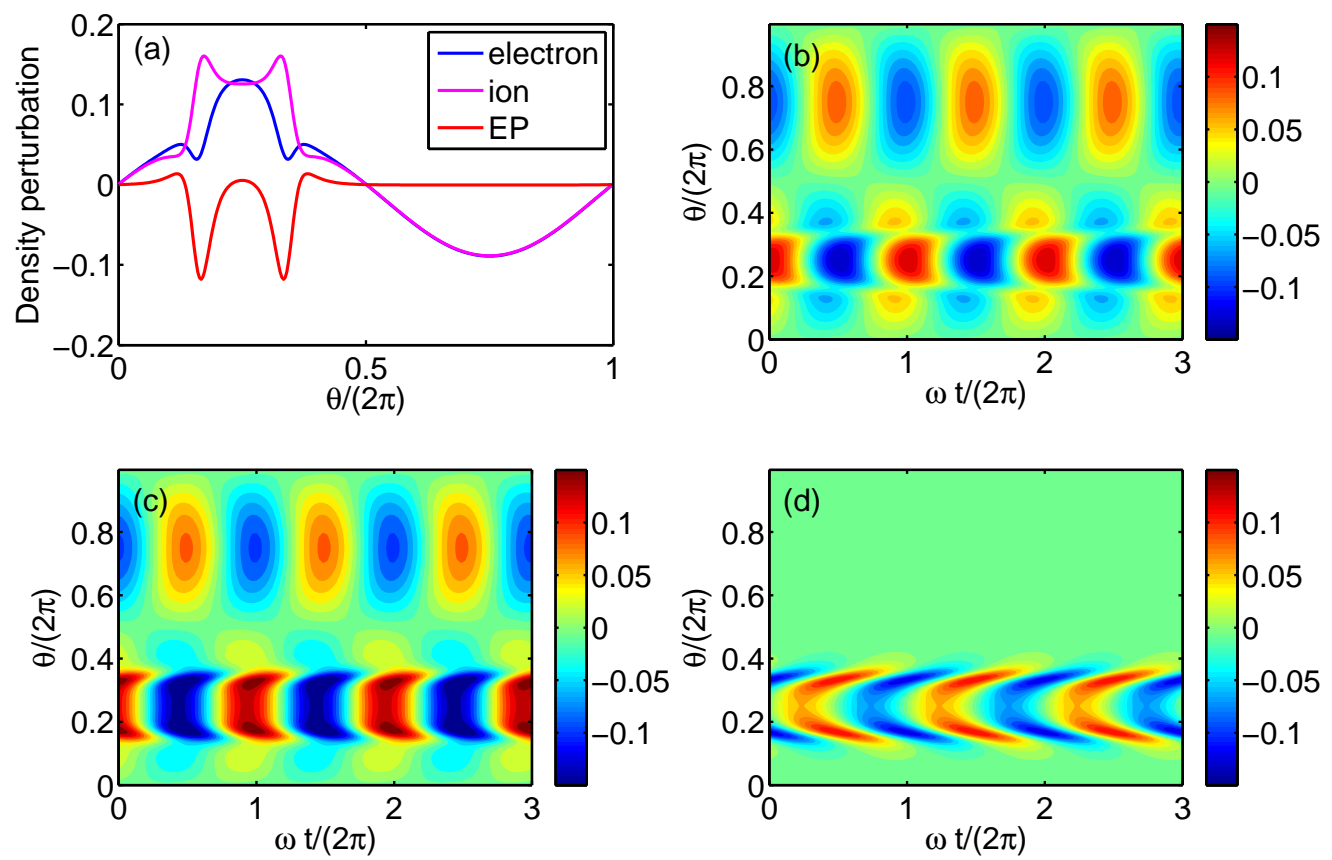

Fig. 3: (a) Real part of the poloidal eigenfunctions for density. The density perturbation of electrons, bulk ions and fast ions are shown. Time evolutions of the density fluctuations of (b) electrons, (c) bulk ions and (d) fast ions, where the time evolution of each species is calculated by $\operatorname{Re}\left[n_{\omega}^{(j)} e^{-i \omega t}\right]$.

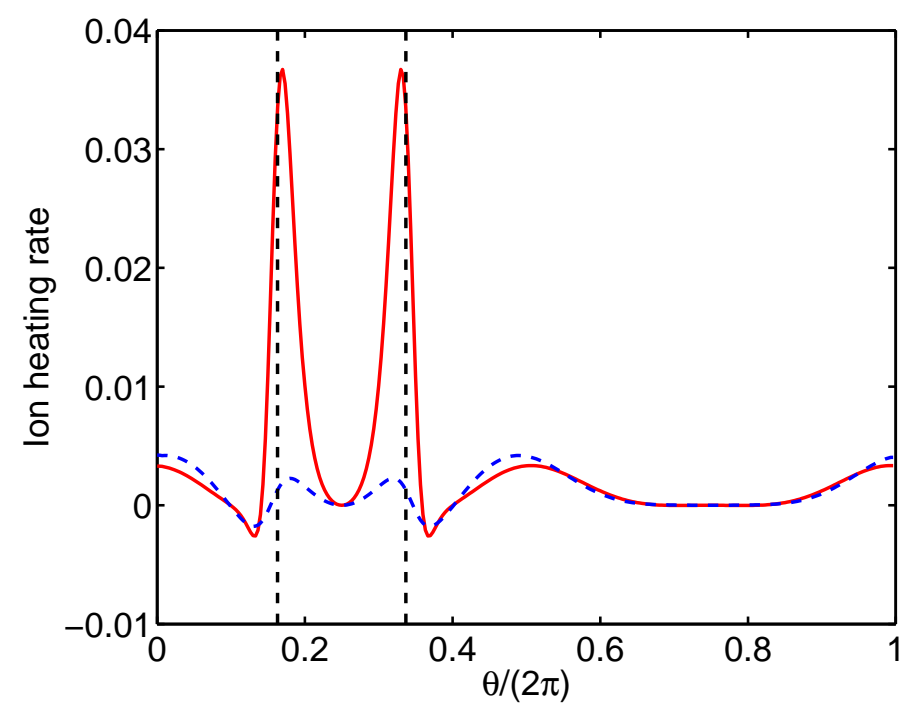

Fig. 4: Poloidal structure of bulk ion heating rate. The dotted line shows the ion heating rate evaluated without the deformation due to the magnetic drift resonance. Here, the heating rate is calculated by using $\phi_{\omega, 0}=1$. The poloidal structure has sharp peaks near the resonance locations. 
$\omega \approx \omega_{D}$ is that the eigenfrequency is proportional to the radial wavenumber. Thus, the radial group velocity is much large compared to the standard GAM branch, and the radial group velocity is comparable to the radial phase velocity as $\partial_{k} \omega \approx \omega / k \sim u_{0}^{2}$. Noting that the unit of this velocity is $v_{T} \rho_{i} / R$. Therefore, the effects of this branch on turbulence and on bulk ion propagate to distant locations in the radial direction. It is noted that there is a report that the turbulence transport increases with the excitation of the EGAM [13]. Thus, detailed researches are necessary to understand such phenomena by considering the nonlocal radial structure of the EGAM and the coupling with turbulence, which is a future work.

\section{Summary}

Eigenmode analysis of EGAMs is performed, based on a set of gyrokinetic equations. Resonance to the magnetic drift of the fast ions is shown to destabilize GAMs. A new branch is found in the family of GAMs, whose frequency is close to the magnetic drift frequency of the fast ions. The poloidal eigenfunction of this branch has bump structures and phase shift in the poloidal direction where the resonance of the magnetic drift with the mode is strong. The ion heating rate by the GAMs is evaluated in the framework of quasi-linear theory. The heating is localized poloidally around the resonance locations. Owing to the bumps in the eigenfunction, the magnitude of the heating rate is much larger than that estimated without the magnetic drift resonance.

\section{Acknowledgement}

This work was partly supported by a grant-in-aid for scientific research of JSPS, Japan (16K18335, 16H02442, 15H02335, 15H02155, 24740372, 21224014) and by the collaboration programs of NIFS (NIFS14KNST072, NIFS15KNST089) and of the RIAM of Kyushu University, and Asada Science foundation.

\section{A Derivation of charge quasi-neutrality}

The derivation of the quasi-neutrality condition, Eq. (3) is explained. The quasi-neutrality condition can be written as $\sum_{j} e_{j}\left\langle\delta f_{r}^{(j)}\right\rangle=0$, where $e_{j}$ is the electric charge of the $j$-th species and $\delta f_{r}^{(j)}$ is the perturbed distribution function written in the real coordinate, and the summation is performed with respect to the electron, bulk ion and fast ions. $\delta f_{r}^{(j)}$ is 
written as [28]

$$
\delta f_{r}^{(j)}=-\phi \partial_{\mathcal{E}} F_{j}+g e^{-i \boldsymbol{k}_{\perp} \cdot \boldsymbol{\rho}},
$$

where $\boldsymbol{\rho}=\boldsymbol{b} \times \boldsymbol{v}$, and $\boldsymbol{b}$ is the unit vector in the direction of the magnetic field. By comparing the expression of $\delta f^{(j)}$ with that of $\delta f_{r}^{(j)}$, the distribution function in the real coordinate can be rewritten as

$$
\delta f_{r}^{(j)}=\delta f^{(j)} e^{-i \boldsymbol{k}_{\perp} \cdot \boldsymbol{\rho}}-\phi \partial_{\mathcal{E}} F_{j}\left(1-J_{0}\left(k_{\perp} v_{\perp}\right) e^{-i \boldsymbol{k}_{\perp} \boldsymbol{\rho}}\right) .
$$

The quasi-neutrality condition can be expressed in the gyro-center coordinate by using Eq. (23) as

$$
\sum_{j} e_{j}\left\langle J_{0}\left(k v_{\perp}\right) \delta f^{(j)}\right\rangle+\sum_{j} e_{j}\left\langle\left(1-J_{0}\left(k v_{\perp}\right)^{2}\right) \partial_{\mathcal{E}} F_{j}\right\rangle=0 .
$$

The second term of Eq. (24) for the bulk ions can be calculated as

$$
\left\langle\left(1-J_{0}\left(k v_{\perp}\right)^{2}\right) \partial_{\mathcal{E}} F_{j}\right\rangle=-\left(1-I_{0}(k) e^{-k}\right),
$$

where $I_{0}(k)$ is the zeroth order modified Bessel function. Equation (3) is obtained by substituting the expression of $\delta f^{(j)}$ into the quasi-neutrality condition. Here, it should be noted that we assume $n_{h} \ll 1$, and we neglect the term corresponding to the polarization density of the fast ions and keep terms related to the resonance.

\section{B Eigenfrequency of the branch with $\omega \sim \omega_{G}$}

The analytical expression of the eigenfrequency of the $\omega_{G}$-branch is derive in order to compare the results reported in $[22,23]$. We considere a situation, $\omega \gg \omega_{D}, q \gg 1$, which is similar condition discussed in $[22,23]$. In this limit, the responses of the bulk ions and the fast ions, Eqs. (11), (15a) can be simplified as

$$
\begin{gathered}
\left\langle J_{0} G_{\omega}^{(i)}\right\rangle \approx\left[\left\langle J_{0}^{2}\right\rangle+\frac{k}{\omega} \sin \theta+\frac{7 k^{2}}{4 \omega^{2}} \sin ^{2} \theta+\cdots\right] \phi_{\omega} \\
\left\langle J_{0} G_{\omega}^{(h)}\right\rangle \approx \frac{2 n_{h} J_{0}^{2} k \omega \sin \theta}{\omega}\left(1+\frac{2 \omega_{D}}{\omega} \sin \theta+\cdots\right) \phi_{\omega} .
\end{gathered}
$$

Combining these responses with Eq. (3), the following relation is obtained.

$$
\phi_{\omega} \approx\left[1+\frac{1}{\tau}\left\{\frac{k}{\omega}+k^{2}\left(\frac{\omega_{G, 0}^{2}}{\omega^{2}} \sin ^{2} \theta-\frac{1}{2}\right)+\frac{2 n_{h} J_{0}^{2} k \omega \sin \theta}{\omega}\left(1+\frac{2 \omega_{D}}{\omega} \sin \theta+\cdots\right)\right\}\right] \phi_{\omega, 0},(28
$$

where $\omega_{G, 0}=\sqrt{7 / 4+1 / \tau}$. Averaging the both side of this relation over the poloidal angle, the following dispersion relation is obtained.

$$
1-\frac{\omega_{G, 0}^{2}}{\omega^{2}}-\frac{4 n_{h} J_{0}^{2} k \omega_{D}}{k^{2} \omega^{2}}=0
$$


Then, the analytical expression for the $\omega_{G}$-branch can be written as

$$
\omega=\sqrt{\omega_{G, 0}^{2}+n_{h} u_{0}^{2} C_{E P}}
$$

where the numerical factor $C_{E P}$ is obtained as $C_{E P}=2 J_{0}^{2}\left(1+\Lambda_{0}^{2}\right)$. The numerical factor becomes $C_{E P}=3 / 2$ in the previous studies such as $[22,23]$, where the distribution of the fast ions are assumed to follow a shifted Maxwellian. The both expressions are similar characteristics. It is noted that the difference of the numerical factors stems from the assumption of the fast ions' distribution function. The dependence of the frequency of the $\omega_{G}$-branch on the density of the fast ions are shown in Fig. 5.

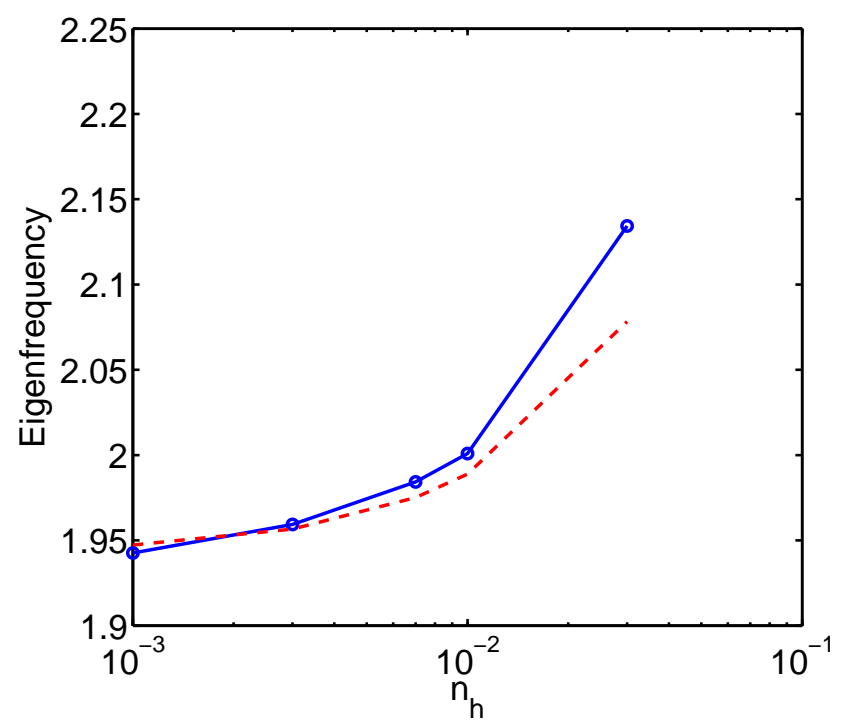

Fig. 5: Dependence of eigenfrequency of $\omega_{G}$-branch against the density of fast ions. The calculation is performed by using the following set of parameters; $q=10, \tau=0.5, k_{r}=$ $0.001, \Lambda_{0}=0.1$. The dashed magenta line corresponds to the analytical expression Eq. (30).

\section{References}

[1] K. Hallatschek, D. Biskamp, Phys. Rev. Lett., 86, 1223 (2001).

[2] P. H. Diamond, S.-I. Itoh, K. Itoh, T. S. Hahm, Plasma Phys. Control. Fusion, 47, R35 (2005).

[3] K. Itoh, K. Hallatschek, S.-I. Itoh, Plasma Phys. Control. Fusion, 47, 451 (2005). 
[4] M. Sasaki, K. Itoh, Y. Nagashima, A. Ejiri, Y. Takase, Phys. Plasmas, 16, 022306 (2009).

[5] R. Hager, K. Hallatschek, Phys. Rev. Lett., 108, 035004 (2012).

[6] G. Y. Fu, Phys. Rev. Lett., 101, 185002 (2008).

[7] H. L. Berk, C.J. Boswell, D. Borba, A.C.A. Figueiredo, T. Johnson, M.F.F. Nave, S.D. Pinches, S.E. Sharapov, JET EFDA contributors, Nucl. Fusion, 46, S888 (2006).

[8] C. J. Boswell, H. L. Berk, D. N. Borba, T. Johnson, S. D. Pinches, S. E. Sharapov, Phys. Lett. A, 358, 154 (2006).

[9] R. Nazikian, G. Y. Fu, M. E. Austin, H. L. Berk, R. V. Budny, N. N. Gorelenkov, W. W. Heidbrink, C. T. Holcomb, G. J. Kramer, G. R. McKee, M. A. Makowski, W. M. Solomon, M. Shafer, E. J. Strait, M. A. Van Zeeland, Phys. Rev. Lett., 101, 185001 (2008).

[10] T. Ido, A. Shimizu, M. Nishiura, S. Nakamura, S. Kato, H. Nakano, Y. Yoshimura, K. Toi, K. Ida, M. Yoshinuma, Nucl. Fusion, 51, 073046 (2011).

[11] T. Ido, M. Osakabe, A. Shimizu, T. Watari, M. Nishiura, K. Toi, K. Ogawa, K. Itoh, I. Yamada, R. Yasuhara, Y. Yoshimura, S. Kato, the LHD Experiment Group, Nucl. Fusion, 55, 083024 (2015).

[12] T. Ido, K. Itoh, M. Osakabe, M. Lesur, A. Shimizu, K. Ogawa, K. Toi, M. Nishiura, S. Kato, M. Sasaki, K. Ida, S. Inagaki, S.-I. Itoh, the LHD Experiment Group, Phys. Rev. Lett., 116, 015002 (2016).

[13] D. Zarzoso, Y. Sarazin, X. Garbet, R. Dumont, A. Strugarek, J. Abiteboul, T. Cartier-Michaud, G. Dif-Pradalier, Ph. Ghendrih, V. Grandgirard, G. Latu, C. Passeron, O. Thomine, Phys. Rev. Lett., 110, 125002 (2013).

[14] D. Zarzoso, X. Garbet, Y. Sarazin, R. Dumont, V. Grandgirard, Phys. Plasmas, 19, $022102(2012)$.

[15] H. Wang, Y. Todo, C. C. Kim, Phys. Rev. Lett., 110, 155006 (2013).

[16] M. Lesur, K. Itoh, T. Ido, M. Osakabe, K. Ogawa, A. Shimizu, M. Sasaki, K. Ida, S. Inagaki, S.-I. Itoh, the LHD Experiment Group, Phys. Rev. Lett., 116, 015003 (2016).

[17] M. Sasaki, K. Itoh, S.-I. Itoh, Plasma Phys. Control. Fusion, 53, 085017 (2011).

[18] T. Ido, M. Osakabe, A. Shimizu, M. Nishiura, T. Itoh, Y. Yoshimura, K. Toi, K. Ogawa, K. Itoh, T. Watari, S. Satake, M. Nakamura, M. Isobe, K. Nagaoka, S. Yamamoto, S. Kato, R. Makino, LHD experiment group, proceedings of IAEA FEC, PD/P8-16 (2012). 
[19] M. Osakabe, T. Ido, K. Ogawa, A. Shimizu, M. Yokoyama, R. Seki, C. Suzuki, M. Isobe, K. Toi, D. A. Spong, K. Nagaoka, Y. Takeiri, H. Igami, T. Seki, K. Nagasaki, LHD experiment group, proceedings of IAEA FEC, EX/10-3 (2014).

[20] Z. Qiu, F. Zonca, L. Chen, Phys. Plasmas, 19, 082507 (2012).

[21] Z. Qiu, F. Zonca, L. Chen, Plasma Phys. Control. Fusion, 52, 095003 (2010).

[22] J. B. Girardo, D. Zarzoso, R. Dumont, X. Garbet, Y. Sarazin, S. Sharapov, Phys. Plasmas, 21, 092507 (2014).

[23] D. Zarzoso, A. Biancalani, A. Bottino, Ph. Lauber, E. Poli, J. B. Girardo, X. Garbet, R.J. Dumont, Nucl. Fusion, 54, 103006 (2014).

[24] K. Miki, Y. Idomura, Plasma and Fusion Res., 10, 3403068 (2015).

[25] H. Wang, Y. Todo, T. Ido, M. Osakabe, Phys. Plamas, 22, 092507 (2015).

[26] H. L. Berk, T. Zhou, Nucl. Fusion, 50, 035007 (2010).

[27] Z. S. Qu, M. J. Hole, M. Fitzgerald, Phys. Rev. Lett., 116, 095004 (2016).

[28] H. Sugama, T.-H. Watanabe, Phys. Plasmas, 13, 012501 (2006).

[29] Z. Gao, K. Itoh, H. Sanuki, J. Q. Dong, Phys. Plasmas, 15, 072511 (2008). 